\title{
Regenerative Failure: A Potential Mechanism for Neuritic Dystrophy in Alzheimer's Disease
}

\author{
David A. DeWitt and J erry Silver \\ Department of Neurosciences, CaseWestern ReserveUniversity, Cleveland, Ohio 44106
}

\begin{abstract}
Although neuronal pathology and synaptic loss are salient features of Alzhei mer's disease (AD), the underlying mechanisms involved are unknown. Using doubleimmunolabeled preparations, we found that both the density and the total lengths of axons are decreased within the A $\beta$-containing area of senile plaques (SP) in comparison with the adjacent neuropil. These observations suggest that axotomy is occurring in the vicinity of the SP which could account for the synaptic loss. Since $A \beta$ in solution has been shown to be neurotoxic in vitro, we tested whether intact SP cores isolated from $A D$ brain were equally detrimental when presented to retinal ganglion neurons. Surprisingly, SPs did not appear to be toxic or even repulsive to neurons since they adhered well and elaborated axons which wrapped tightly around the SP core. In the presence of cortical astrocytes, however, neurons appeared to avoid SP cores. We found that astrocytes accumulate and deposit chondroitin sulfate proteoglycans (CSPGs) around SP cores in vitro in a pattern similar to that observed around SPs in Alzheimer's disease brain. Neuronal avoidance of astrocyte-conditioned SP cores could be due to the axon outgrowth inhibitory nature of CSPGs. These results suggest that astrocytic reaction to SPs, including increased CSPGs, may facilitate the decreased axon density and synaptic loss in AD brain. Moreover, the similarities between swollen axon endings following axotomy in trauma and the dystrophic neurites of the SP suggest that dystrophic neurites in $A D$ may be exhibiting regenerative failure rather than aberrant sprouting. $\odot 1996$ Academic Press, Inc.
\end{abstract}

\section{INTRODUCTION}

Senile plaques (SPs) of Alzheimer's disease are cellularly and molecularly complex structures. Reactive astrocytes $(5,21,28,30)$, activated microglia $(14,17$, 24), and dystrophic neurites are present in SPs. In addition to $A \beta$, over 40 protein components have been identified which accumulate in the SP including proteases (30), protease inhibitors $(1,11,18)$, growth factors $(12,39)$, and extracellular matrix proteins (16) including proteoglycans $(9,32-35)$. How all of the various SP components interact in the pathogenesis of Alzheimer's disease (AD) is far from clear.

Alzheimer's disease pathology includes loss of specific neuronal populations, synapseloss, senile plaques, and neurofibrillary tangles. While both SPs and neurofibrillary tangles are inexorably linked with Alzheimer's disease, synaptic loss shows the strongest linkage to severity of dementia $(23,38)$. Although global loss of synapses occurs in the brains of individuals with Alzheimer's disease, synapse loss is accentuated within core containing senile plaques (22). Dystrophic neurites, found around SPs, are swollen neuronal processes which contain the same abnormal cytoskeletal el ements and accumulated proteins found in neurofibrillary tangles (8, 13, 19, 21, 27). The mechanism(s) involved in synaptic loss and induction of neuritic dystrophy has not been defined, although $A \beta$ has been suggested by many to underlie the neuronal pathology.

Numerous studies have suggested that $A \beta$ is neurotoxic in vitro; however, most studies of in vivo brain injections of $A \beta$ have not shown consistent neurotoxicity (31). When synthetic $A \beta$ is presented as a substrate, neurons adhere and elaborate axons normally $(5,20)$. Further, when neurons were grown on cryostat sections from Alzheimer's disease brain, they readily adhered and survived even on SPs (6). Interestingly, these neurons avoided the senile plaque periphery, an area which is rich in chondroitin sulfate proteoglycans (9). Nonetheless, the demonstration of $A \beta$ toxicity in solution (i.e., neuronal death) is not equivalent to $A \beta$ induction of neuritic dystrophy (i.e., a living neuron with swollen, torturous neurites), which to our knowledge has not yet been shown.

One hypothesis that has been put forth to explain neuritic dystrophy is that it is caused by aberrant sprouting (10). In such a scenario, an increased amount of growth factors and growth-promoting extracellular matrix molecules in the SP vicinity is suggested to provide a strong trophic signal to neurons, causing them to sprout abnormally. If this were true, one might expect to find an increase in the density or total length of axons within the SP area. In the present study, we have tested this hypothesis using double immunolabeled partial reconstructions to determine the density 
and lengths of axons within the $A \beta$-containing area of the SP. In this study, we present the hypothesis that synapse loss and possibly neuritic dystrophy in the SP are the result of a process of regenerative failureakin to that which occurs following axotomy in the adult CNS due to trauma.

\section{MATERIALS AND METHODS}

\section{Axon Density/ Length Analysis}

Sections from frontal cortex or hippocampus from three confirmed cases of Alzheimer disease were studied. Tissue was fixed in methacarn (methanol 6: chloroform 3: acetic acid 1) and embedded in paraffin prior to sectioning. 3',3'-Diaminobenzidine was used as the cosubstratewith the peroxidase anti-peroxidasemethod for immunostaining (37). SPs were verified by Congo red or RGP-9, an antisera to A $\beta$ (gift of George Perry, Case Western Reserve University). Normal neurites were identified with SMI-31, a monoclonal antibody to phosphorylated neurofilament. This antibody also stains dystrophic neurites which can be identified by their size and shape.

In order to determine axon density and total length within SPs, double-immunolabeled preparations were stained with SMI-31 and RGP-9. SMI-31 was visualized using DAB while RGP-9 was visualized using alkaline phosphatase as previously described (9). Up to four adjacent 10- $\mu \mathrm{m}$ sections were immunostained in order to verify that a particular SP was a corecontaining plaque. Following verification, the area occupied by $A \beta$ was determined using the Quantimet image analysis system with the 40X objective. The number, lengths, and widths of axons within the total area occupied by $A \beta$ in core-containing plaques were also determined using the Quantimet system. In some cases, paired measures of non-A $\beta$-containing control areas were also obtained. In this case, the stage was moved to a random field within $150 \mu \mathrm{m}$ of the SP and within the same brain layer. The number, lengths, and widths of axons in the normal neuropil in an area equivalent to the senile plaque were determined. Total axon length was determined by summing the lengths of all axons within the specified areas.

\section{I solation of SenilePlaqueCores}

SP cores were isolated as previously described (29). Briefly, portions of frontal and temporal lobe from individuals with confirmed, severeAlzheimer's disease, were obtained at autopsy and frozen. Gray matter was homogenized and brought to $97^{\circ} \mathrm{C}$ for $10 \mathrm{~min}$ in $2 \%$ SDS, $1 \mathrm{mM}$ Tris (pH 7.6), sieved through 110- $\mu$ m nylon mesh, and centrifuged at $300 \mathrm{~g}$ for $30 \mathrm{~min}$ to pellet the core-containing fraction. The samples werethen washed in $0.1 \% \mathrm{SDS}, 150 \mathrm{mM} \mathrm{NaCl}, 0.02 \% \mathrm{NaN}_{3}$ and centrifuged again at $300 \mathrm{~g}$ for $10 \mathrm{~min}$. The samples were sieved through a 35- $\mu$ m nylon mesh and then applied to a noncontinuous sucrose gradient $(1.2,1.4,1.6$, and 1.8 $M$ sucrose) and centrifuged again at $72,000 \mathrm{~g}$ for $60 \mathrm{~min}$. M ost of the SP cores were collected from the 1.4-1.6 M interface. SP cores were stored at $4^{\circ} \mathrm{C}$ until use.

\section{Neuronal Cultures}

Chick retinal ganglion neurons were obtained by carefully dissecting the retina from stage 29 or 30 ( E 6 or E 7) chick embryos and briefly dissociating the tissue in trypsin. The neurons were plated on poly-L-lysinecoated coverslips and grown in DMEM-F 12 medium (Gibco) with insulin, transferin, sodium selenate supplement (Sigma), and only $1 \%$ fetal calf serum. Neurons were grown on coverslips which previously had up to 500 isolated SP cores dried to the poly-L-lysine-coated substrate. In some cases, the SP cores were coated with laminin to promote robust neurite outgrowth.

\section{AstrocyteCultures/ Neuronal Co-cultures}

Astrocytes were obtained from the cortex of P0 Sprague-Dawley rat pups. Mixed glial cultures were grown on poly-L-lysine-coated flasks with DMEM-F 12 (Gibco) and 10\% fetal calf serum. After $24 \mathrm{~h}$, microglia and other cell types were removed through shaking. The remaining astrocytes were used after 2 weeks in culture. About $5 \times 10^{4}$ cells per well were grown with SP cores for 1-2 weeks prior to the addition of neurons. Astrocyte-conditioned SP cores were also processed for immunocytochemistry.

\section{I mmunocytochemistry}

I mmunocytochemistry followed the method of Sternberger (37). Coverslips were fixed in $2 \%$ paraformaldehyde in $1 \mathrm{M}$ PBS, $\mathrm{pH}$ 7.2, for $1 \mathrm{~h}$ and rinsed with $1 \mathrm{M}$ PBS. Endogenous peroxidase activity was blocked with $3 \%$ hydrogen peroxide for $30 \mathrm{~min}$ followed by a 30-min incubation in saturated Congo red solution (70\% ethanol, saturated with $\mathrm{NaCl}$ ) to aid in the identification of SP cores. The coverslips were further blocked with $10 \%$ normal goat serum for 30 min prior to the addition of primary antibodies. SP core material was verified with the monoclonal $4 \mathrm{G} 8$ or polyclonal antisera raised to $A \beta$. Primary antibodies GFAP (astrocyte marker), CS-56 (chondroitin sulfate glycosaminoglycan) (7), and TUJ 1 (neuronal marker) were incubated on the coverslips overnight at $4^{\circ} \mathrm{C}$. Goat vs mouse or goat vs rabbit secondaries were applied with fluorescent tagged or peroxidase anti-peroxidase as tertiary antibodies. Diaminobenzidine was used as chromagen with peroxidase-conjugated antibodies. 


\section{RESULTS}

\section{Axonal Loss in SenilePlaques}

Sections through the hippocampus from individuals with Alzheimer's disease which have been immunolabeled for neurofilament protein contain large numbers of "axon holes" where the SP core is located. Anti- neurofilament-stained preparations show axon clearings in the area of the SP (Figs. $\mathrm{AA}$ and $1 \mathrm{C}$ ) which is demonstrated in the adjacent sections (Figs. $1 \mathrm{~B}$ and 1D). In order to quantitate the extent of axonal loss, axon density and total axon length were determined for the entire A $\beta$-containing area of the SP. These results are summarized in Table 1, which confirms that the

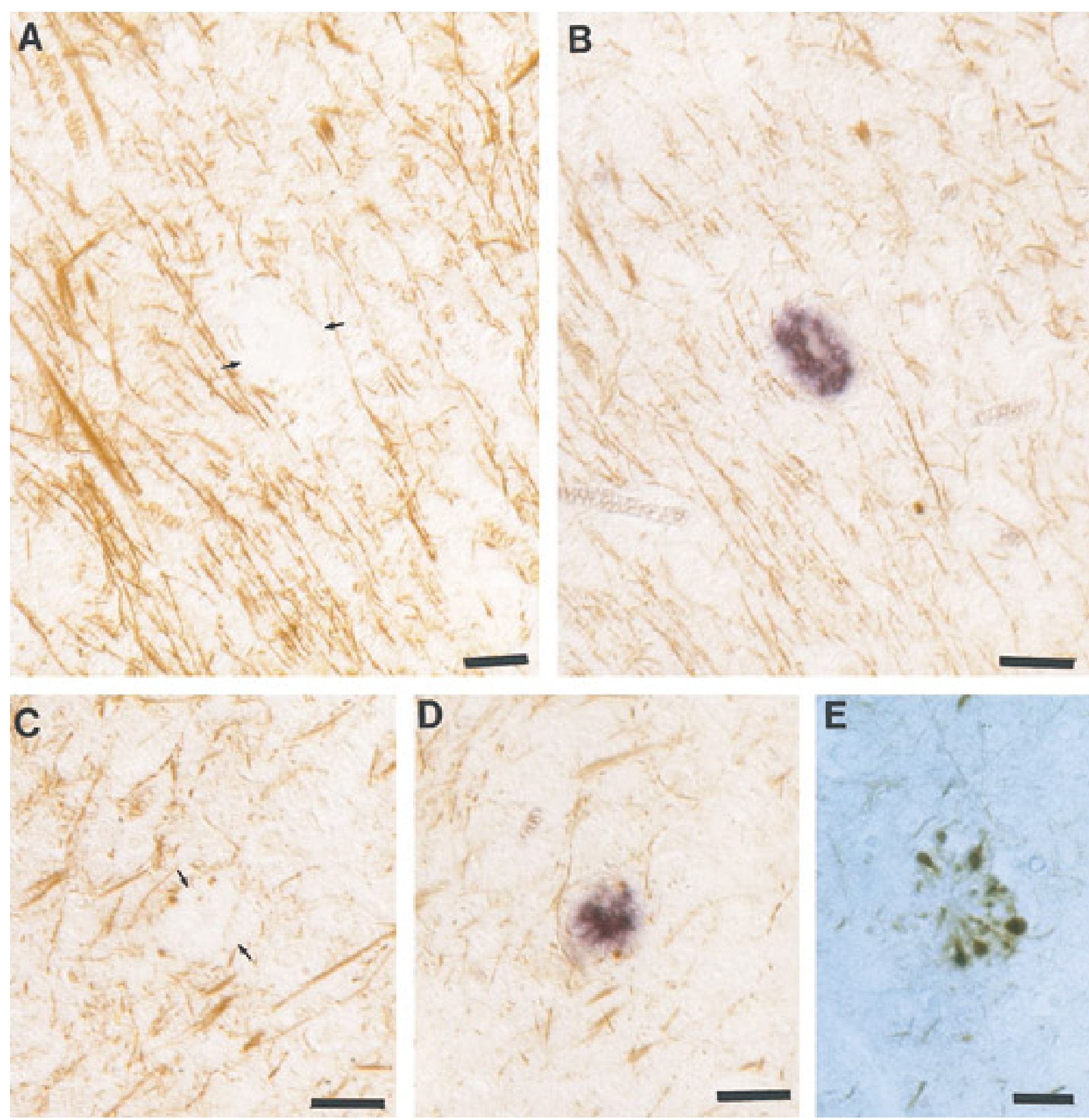

FIG. 1. Antibody to neurofilament protein (SMI-31) reveals axonal staining as well as "axonal holes" (arrows) in the area of a senile plaque $(A, C)$. The adjacent sections double-immunolabeled for A $\beta$ (RGP-9, purple) demonstrate that the axonal hole is within the $A \beta$-containing area of the senile plaque (B,D). Dystrophic neurites are generally found in a radial pattern around the senile plaque (E). Scale bars, $20 \mu \mathrm{m}$. 


\section{TABLE 1}

Neurite Analysis-Comparison of the Density and Total Length of Axons within the A $\beta$-Containing Area of a Core Containing Senile Plaque and Areas without $A \beta$

\begin{tabular}{lll}
\hline & Normal neuropil & \multicolumn{1}{c}{ Senile plaque } \\
\hline Total area & $3033 \mu \mathrm{m}^{2}$ & $9113 \mu \mathrm{m}^{2}$ \\
No. neurites/area & $13.8 \mathrm{~N} / 1000$ & $4.4 \mathrm{~N} / 1000$ \\
& $\mu \mathrm{m}^{2} \pm 2.76 \mathrm{SE}$ & $\mu \mathrm{m}^{2} \pm 1.4 \mathrm{SE}$ \\
Average neurite length & $9.14 \mu \mathrm{m} \pm 0.89 \mathrm{SE}$ & $7.23 \mu \mathrm{m} \pm 0.69 \mathrm{SE}$ \\
Average neurite width & $1.04 \mu \mathrm{m} \pm 0.11 \mathrm{SE}$ & $1.48 \mu \mathrm{m} \pm 0.12 \mathrm{SE}$ \\
Neurite length per area & $126 \mathrm{~mm} \mathrm{~N} / \mathrm{mm}^{2}$ & $32.6 \mathrm{~mm} \mathrm{~N} / \mathrm{mm}^{2}$ \\
\hline
\end{tabular}

density of axons within SP is significantly decreased in comparison with the adjacent neuropil. Moreover, the total length of all axon segments within SPs was also substantially lower than the adjacent area lacking $A \beta$. These results are consistent with the hypothesis that $A \beta$ or other components present in the SP lead to physiological or de facto axotomy or axon retraction.

\section{Neurons Elaborate Axons on SenilePlaqueCores in Vitro}

Since numerous in vitro studies have suggested that aggregated $A \beta$ in solution is neurotoxic, this protein seemed a likely candidate responsible for axonal loss in vivo. In order to determine whether $A \beta$ in the context of an intact SP might be responsible for axotomy, we developed a novel model in which SPs isolated from AD brain are presented to neurons. By drying the SPs to the coverslips prior to addition of neurons, the $A \beta$ is presented as a 3-dimensional, aggregated, chronic, and focal source much as it may be in vivo. This presentation of $A \beta$ allowed individual neurons to "choose" whether or not to interact with the SP. I nitially, SP cores and the poly-L-lysine substrate were coated with laminin to facilitate neuronal adhesion as well as to promote neurite outgrowth. Neurons grew in close proximity to SP cores and wrapped axons around them. Since thelaminin coating may have hindered detrimental effects of $A \beta$, neurons were also grown on SP cores without laminin. In this case, neurons still adhered to SP cores and elaborated axons toward and tightly hugging the core (Fig. 2E).

\section{Astrocytes Accumulate CSPGs Which May Inhibit Axon Outgrowth}

Astrocytes show increased GFAP immunoreactivity in association with SPS in AD brain (Fig. 2A). This increase is paralleled in vitro when rat cortical astrocytes are grown on isolated SP cores (Fig. 2C). We have previously shown that astrocytes deposit increased amounts of CSPGs in the extracellular matrix when grown on a synthetic $A \beta$ peptide substrate (5). Therefore, we asked whether astrocytes might also accumulate or deposit CPSGs in response to isolated SP cores. Indeed, we found that astrocytes in contact with SP cores show increased CSPG immunoreactivity around the core periphery (Fig. 2D). CSPG accumulated in a ring-like pattern just as observed in AD brain (Fig. 2B).

\section{Neurons Avoid Astrocyte-Conditioned Senile PlaqueCores}

Since certain CSPGs have been found to inhibit axon outgrowth in vitro $(5,25,36)$, we asked whether the response of astrocytes to SP cores might alter the usual neuronal response of growing in intimate contact with the SP cores. Astrocytes were allowed to react to SP cores for 1-2 weeks prior to addition of neurons. Indeed, following astrocyte conditioning of bound SP cores, axons appeared to grow further from the SP core perimeter and in some cases appeared to avoid the astrocyte-conditioned SP cores altogether (Fig. 2F). These results are consistent with the possibility that astrocytes deposit an axon repulsive extracellular matrix in response to SPs.

\section{DISCUSSION}

In this study we have demonstrated that both the density of axons as well as thetotal lengths of axons are substantially decreased within the $A \beta$-containing area of the SP. However, these results alone, while consistent with axotomy within the SP, do not help to clarify which factors are responsible for axonal loss or neuritic dystrophy. Since $A \beta$ has been suggested to be neurotoxic, we examined the effects of SP cores, isolated from the cortex of individuals with Alzheimer's disease, on neurons in vitro. Surprisingly, neurons adhered to SP cores and elaborated axons which wrapped tightly around the core surface, suggesting that factors other

FIG. 2. In AD brain, astrocytes are hypertrophied and show increased GFAP immunoreactivity (brown) in association with a senile plaque (c) core (A). CSPG immunoreactivity reveals an increase in the vicinity of the senile plaque periphery (B) which partially overlaps GFAP. Rat cortical astrocytes growing on SP cores in vitro show increased GFAP immunoreactivity (C) and CSPG immunoreactivity (D) (CS-56, brown) immediately adjacent to the senile plaque (c) core. The pattern of GFAP and CSPG immunoreactivity in vitro is similar to that found around senile plaques in $A D$ brain (compare $A$ and $B$ with $C$ and $D$ ). Retinal ganglion neurons grew in close association with senile plaque (c) cores isolated from AD brain (E, arrow). When neurons were grown on astrocytes which had reacted to SP cores for 2 weeks, the neurons avoided the area of the SP and axons appeared to curve away (F, arrows). Scale bars, $20 \mu \mathrm{m}$. 

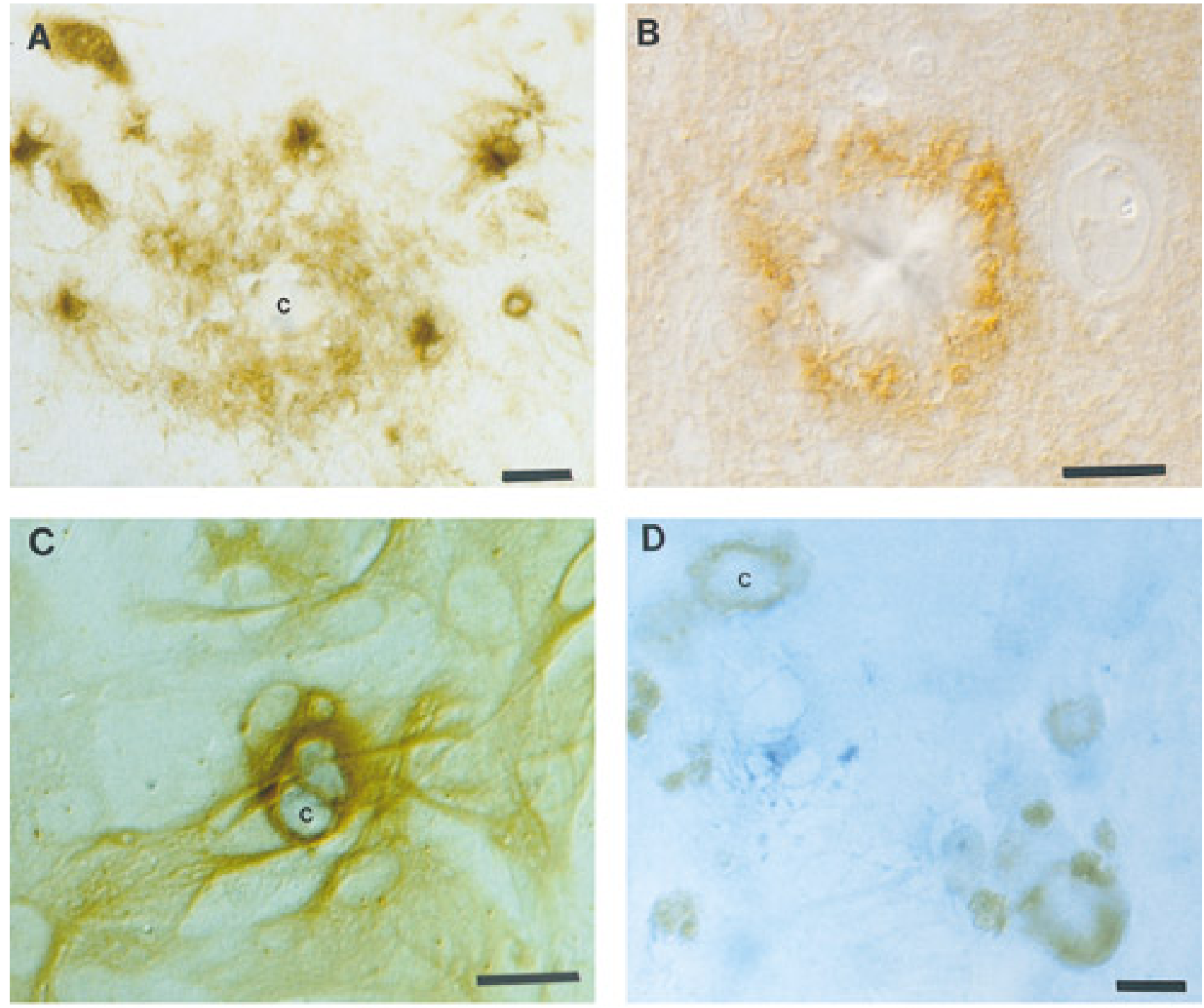

\section{E}

F

2

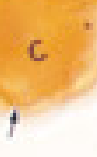


than or in addition to $A \beta$ may be involved in the axonal loss in vivo. However, when neurons were grown on astrocytes responding to SP cores, axons now avoided such conditioned SP cores altogether. These results are consistent with the possibility that enhanced deposition of axon inhibitory CSPGs, or other sulfated proteoglycans, by reactive astrocytes could be an additional factor promoting neuronal pathology in the SP.

Previously, Benes et al. (2) compared the axon density within and immediately outside the core of SPs. They found that the axon density was decreased within the core and up to two plaque core distances away and argued that if the mere presence of $A \beta$ pushed axons out (or induced excessive sprouting), then there would be an increased density immediately outside the core. Since this does not occur, they suggested that there was axonal loss within and adjacent to the SP core, which would be consistent with axotomy. We have confirmed and extended these findings to include a decrease in the total length of axons within the SP.

Additional evidence suggests that the periphery of the SP, rather than the core itself, may have the greatest impact on neurons. Carpenter et al. (6) grew neurons on cryostat sections from AD brain. There was no difference in the density of neurons which adhered to SP cores and those which adhered to areas lacking SPs. However, the number which adhered to the periphery of the SP was significantly reduced. F urther, neurons growing in the peri-plaque region showed inhibited axon outgrowth and the axons appeared to avoid this area. These results, along with the finding of decreased axon density in SPs in vivo, strongly suggest that a factor(s) within the SP periphery exerts an inhibitory effect on axon outgrowth.

A likely factor involved could be proteoglycans. Previously, we localized CSPG accumulation to the periphery of SPs - the same area shown to inhibit axon outgrowth in the cryostat model (6). In addition, we (9) found that astrocytes respond to a synthetic $A \beta$ substrate with the deposition of axon repulsiveamounts or types of CSPGs (5). The results of the present study provide further evidence that the accumulation of CSPGs by astrocytes in response to SP may, indeed, have detrimental (i.e., barrier) effects on neurons.

Is it possible that CSPGs and/or reactive astrocytes in addition to forming a barrier to regeneration also promote neuronal dystrophy in the first place? The first evidence in support of this has come from a recent paper by Holtmatt and colleagues (15) who examined primary sensory olfactory axons in adult animals genetically engineered to overproduce GAP-43. When these axons are compelled to regenerate into areas around the glomerulus that are rich in reactive astrocytes and CSPG, they develop enlarged, paddle-shaped, dystrophic endings and varicosities. Although the authors did not mention Alzheimer's disease in this paper, these unusual axon endings bear striking similarities to the dystrophic neurites of the SP as well as the sterile endings described following trauma (4). Such truly dystrophic neurites have yet to be generated in vitro in response to $A \beta$ or proteoglycans, perhaps because only embryonic neurons have been used or because of an inappropriate 3-dimensional presentation. However, the spherical nature of the peri-glomerular region and its striking similarity to the penumbra of the SP may enable us to begin to understand how neuronal dystrophy, reactive astrocytes, and proteoglycans are interrelated.

The aberrant sprout hypothesis of neuritic dystrophy (10) has received much support because of the many growth factors and growth-promoting molecules which have been found in SPs including bFGF (12), laminin, and collagen (26). Although it has been suggested that excessive or abnormal sprouting of neurites due to increased trophism may cause neuritic dystrophy, the relation of trophic molecules to regeneration has not been overlooked (22). I ndeed, GAP43 and other growthpromoting/associated molecules which are present in SPs are also characteristic of wound- or regenerationrelated events.

This suggests a different view of the abnormal neurites and extracellular matrix proteins in SPs. Our morphometric analysis of AD tissue raises the possibility that instead of a totally growth-promoting, trophic environment for excessive neurite outgrowth, the swollen nature of the dystrophic neurites suggests that the environment of the SP periphery may prevent the restoration of synapses by inhibiting neuritic sprout infiltration into the area. Such an idea has support since the bulbous, dystrophic neurites found in SPs of $A D$ brain differ from degenerating neurites found in other neurological conditions (3). These other neurodegenerative diseases are not characterized by the large, focal extracellular deposits of wound-related molecules which occur in Alzheimer's disease. Thus, we suggest that the dystrophic neurites of Alzheimer's disease are remarkably similar to the abortive sprouts with sterile clubs classically described by Cajal which occur in regenerative failure (4). Therefore, we propose that the dystrophic neurites in $A D$ are undergoing abortive regeneration rather than aberrant sprouting.

\section{ACKNOWLEDG MENTS}

This work was supported by NIH Grant NS25713. D. DeWitt was also supported by predoctoral fellowship AG-00105-11A1. The authors acknowledge P. Gambetti for use of the Quantimet image analysis system. The authors gratefully acknowledge the technical assistance of Cathy Doller.

\section{REFERENCES}

1. Abraham, C. R., D. J. Selkoe, and H. Potter. 1988. I mmunohistochemical identification of the serum protease inhibitor a1- 
antichymotrypsin in the brain amyloid deposits of Alzheimer's disease. Cell 52: 487-501.

2. Benes, F. M., P. A. Farol, R. E. Majocha, C. A. Marotta, and E. D. BIRD. 1991. Evidence for axonal loss in regions occupied by senile plaques in Alzheimer cortex. Neuroscience 42: 651-660.

3. Benzing, W. C., E. J. Mufson, and D. M. Armstrong. 1993. Alzheimer's disease-like dystrophic neurites characteristically associated with senile plaques are not found within other neurodegenerative diseases unless amyloid $\beta$-protein deposition is present. Brain Res. 606: 10-18.

4. CAJ AL, R. S. 1928. Degeneration and Regeneration of theNervous System. Hafner, New York.

5. Canning, D. R., R. J . McKeon, D. A. DeWitt, G. Perry, J . Wuj ek, R. FREDRICKSON, AND J. SILVER. 1993. $\beta$-Amyloid of Alzheimer's disease induces functionally reactive gliosis. Exp. Neurol. 124: 289-298.

6. Carpenter, M. K., K. A. Crutcher, and S. B. Kater. 1993. An analysis of the effects of Alzheimer's plaques on living neurons. Neurobiol. Aging 14: 207-215.

7. Couchman, J . R., B. Caterson, J . E. Christner, and J . R. Baker. 1984. Mapping by monoclonal antibody detection of glycosaminoglycans in connective tissues. Nature 307: 650-652.

8. Cras, P., M. Kawal, D. Lowery, P. Gonzallez-DeWhitt, B. Greenberg, and G. Perry. 1991. Senile plaque neurites in Alzheimer disease accumulate amyloid precursor protein. Proc. Natl. Acad. Sci. USA 88: 7552-7556.

9. DeWitt, D. A., J. Silver, D. R. Canning, and G. Perry. 1993. Chondroitin sulfate proteoglycans are associated with the le sions of Alzheimer's disease. Exp. Neurol . 121: 149-152.

10. Gedes, J. W., K. J. Anderson, and C. W. Cotman. 1986. Senile plaques as aberrant sprout-stimulating structures. Exp. Neurol . 94: 767-776.

11. Gollin, P. A., R. N. Kalaria, P. Eikelenboom, A. Rozemuller, AND G. PERRY. 1992. Alpha 1-antitrypsin, and alpha 1-antichymotrypsin are in the lesions of Alzheimer's disease. Neuroreport 3: 201-203.

12. Gomez-Pinilla, F., B. J . Cummings, and C. W. Cotman. 1990. Induction of basic fibroblast growth factor in Alzheimer's disease pathology. Neuroreport 1: 211-214.

13. Grundke-I qbal, I., K. I qbal, Y. C. Tung, M. Quinlan, H. M. WISNIEWSKI, AND L. I. BINDER. 1986. Abnormal phosphorylation of the microtubule-associated protein $\tau$ (tau) in Alzheimer cytokeletal pathology. Proc. Natl. Acad. Sci. USA 83: 49134917.

14. HaGA, S., K. AKAI, AND T. I SHII. 1989. Demonstration of microglia cells in and around senile (neuritic) plaques in the Alzheimer brain: An immunohistochemical study using a novel monoclonal antibody. Acta Neuropathol. 77: 569-575.

15. Holtmatt, A. J . G. D., P. A. Dij khuizen, A. B. Oestreicher, H.J . Romij N, N. M. T. VAN Der Lugt, A. Berns, F. L. Margolis, W. H. GISPEN, AND J. VerhaAgen. 1995. Directed expression of the growth-associated protein B-50/GAP 43 to olfactory neurons in transgenic mice results in changes in axon morphology and extraglomerular fiber growth. J . Neurosci. 15: 7953-7965.

16. HowARD, J ., AND G. J . PILKIngton. 1990. Antibodies to fibronectin bind to plaques and other structures in Alzheimer's disease and control brain. Neurosci. Lett. 118: 71-76.

17. Itagaki, S., P. L. McGeer, H. Akiyama, S. Zhu, and D. Selkoe. 1989. Relationship of microglia and astrocytes to amyloid deposits of Alzheimer disease. J . Neuroimmunol . 24: 173-182.

18. KALARIA, R. N. 1993. Cerebral microvasculatureand immunological factors in Alzheimer's disease. Clin. Neurosci. 1: 204-211.

19. Kawal, M., P. Cras, P. Richey, M. Tabaton, D. E. Lowery, P. A. Gonzalez-DeWhitt, B. D. Greenberg, P. Gambetti, and G.
PERRY. 1992. Subcellular localization of amyloid precursor protein in senile plaques of Alzheimer's Disease. Am. J . Pathol. 140: 947-958.

20. Koo, E. H., L. Park, and D. J. Selkoe. 1993. Amyloid $\beta$-protein as a substrate interacts with extracellular matrix to promote neurite outgrowth. Proc. Natl. Acad. Sci. USA 90: 4748-4752.

21. Mandybur, T. I., And C. C. Chuirazzi. 1990. Astrocytes and the plaques of Alzheimer's disease. Neurology 40: 635-639.

22. Masliah, E., M. Mallory, L. Hansen, M. Alford, R. Deteresa, R. Terry, J . BAUdiER, AND T. SAITOH. 1992. GAP43-immunoreactive aberrant sprouting neurites in Alzheimer's disease. Brain Res. 574: 312-316.

23. Masliah, E., R. Terry, M. Mallory, M. Alford, and L. Hansen. 1990. Diffuse plaques do not accentuate synapse loss in Alzheimer's disease. Am. J . Pathol. 137: 1293-1297.

24. McGeer, P. L., S. Itagaki, H. Tago, and E. G. McGeer. 1987. Reactive microglia in patients with senile dementia of the Alzheimer type are positive for the histocompatibility glycoprotein HLA-DR. Neurosci. Lett. 79: 195-200.

25. MCKeon, R. J ., R. C. Schreiber, J . S. Rudge, ANd J . Silver. 1991. Reduction of neurite outgrowth in a model of glial scarring following CNS injury is correlated with the expression of inhibitory molecules on reactive astrocytes. J. Neurosci. 11: 3398-3411.

26. Perlmutter, L. S., E. Barron, D. Saperia, and H. C. Chui. 1991. Association between vascular basement membrane components and the lesions of Alzheimer's disease. J . Neurosci. Res. 30: 673-681.

27. Perry, G., R. Friedman, G. Shaw, and V. Chau. 1987. Ubiquitin is detected in neurofibrillary tangles and senile plaque neurites of Alzheimer disease brains. Proc. Natl. Acad. Sci. USA 84: 3033-3036.

28. Pike, C. J., B. J. Cummings, and C. W. Cotman. 1995. Early association of reactive astrocytes with senile plaques in Alzheimer's disease. Exp. Neurol. 132: 172-179.

29. Selkoe, D. J ., C. R. Abraham, M. B. Podlisny, and L. K. Duffy. 1986. I solation of low-molecular-weight proteins from the amyIoid plaque fibers in Alzheimer's disease. J . Neurochem. 46: 1820-1834.

30. Smith, M. A., R. N. Kalaria, and G. Perry. 1993. $\alpha_{1}$-Trypsin immunoreactivity in Alzheimer disease. Biochem. Biophys. Res. Commun. 193: 579-584.

31. Snow, A. D. AND A. T. MaLouf. 1993. In vitro and in vivo models to unravel the potential roles of $\beta / A 4$ in the pathogenesis of Alzheimer's disease. Hippocampus 3: 257-268.

32. Snow, A. D., H. Mar, D. Nochlin, H. Kresse, and T. N. Wight. 1992. Peripheral distribution of dermatan sulfate proteoglycans (Decorin) in amyloid-containing plaques and their presence in neurofibrillary tangles of Alzheimer's disease. J . Histochem. Cytochem. 40: 105-113.

33. Snow, A. D., H. Mar, D. Nochlin, R. T. Sekiguchi, K. Kimata, Y. KoILE, AND T. N. Wight. 1990. Early accumulation of heparan sulfate in neurons and in the beta-amyloid protein-containing lesions of Alzheimer's disease and Down's syndrome. Am. J . Pathol . 137(5): 1253-1270.

34. Snow, A. D., D. Nochlin, R. Sekiguchi, and S. S. Carlson. 1996. Identification and immunolocalization of a new class of proteoglycan (keratan sulfate) to the neuritic plaques of Alzheimer's disease. Exp. Neurol. 138: 305-317.

35. Snow, A. D., J. P. Willmer, AND R. KisileVsky. 1987. Sulfated glycosaminoglycans in Alzheimer's disease. Hum. Pathol . 18(5): 505-510.

36. Snow, D. M., V. Lemmon, D. A. Carrino, A. I. Caplan, and J . SILVER. 1990. Sulfated proteoglycans in astroglial barriers inhibit neurite outgrowth in vitro. Exp. Neurol. 109: 111-130. 
37. Sternberger, L. A. 1986. Immunocytochemistry. 3rd ed. Wiley. New York.

38. Terry, R. D., E. Masliah, D. P. Salmon, N. Butters, R. DeTeresa, R. Hill, L. A. Hansen, and R. Katzman. 1991. Physical basis of cognitive alterations in Alzheimer disease: Synapse loss is the major correlate of cognitive impairment. Ann. Neurol. 30: 572-580.

39. van der Wal, E. A., F. Gomez-Pinilla, and C. W. Cotman. 1993. Transforming growth factor- $\beta 1$ is in plaques in Alzheimer's and Down's pathologies. Neuroreport 4: 69-72. 